\title{
Discussion
}

Public Health Genomics
Public Health Genomics 2019;22:145-161

DOI: $10.1159 / 000504785$
Received: November 5, 2019

Accepted: November 14, 2019

Published online: December 13, 2019

\section{Artificial Intelligence: Power for Civilisation - and for Better Healthcare}

\author{
Denis Horgan $^{a}$ Mario Romao ${ }^{b}$ Servaas A. Morréc, d Dipak Kalra $^{e}$ \\ ${ }^{a}$ European Alliance for Personalised Medicine, Brussels, Belgium; ${ }^{b}$ Intel, Brussels, Belgium; ${ }^{C}$ Institute for Public \\ Health Genomics, Department of Genetics and Cell Biology, Research Institute GROW, Faculty of Health, Medicine \\ and Life Sciences, University of Maastricht, Maastricht, The Netherlands; ${ }^{d}$ Laboratory of Immunogenetics, \\ Department of Medical Microbiology and Infection Control, VU University Medical Center, Amsterdam, \\ The Netherlands; ${ }^{\mathrm{e}}$ The European Institute for Innovation through Health Data (i HD), Gent, Belgium
}

\section{Keywords}

Artificial intelligence $\cdot$ Big data $\cdot$ Regulatory framework .

Member States · Systems · Enablers · Innovation .

Commission · European Union · Genomics · Machine

learning $\cdot$ Information and communication technology

Digital health . Personalised medicine - Precision medicine .

Personalised healthcare · Diagnostics · Information · Value

\begin{abstract}
Artificial intelligence (AI) is changing the world we live in, and it has the potential to transform struggling healthcare systems with new efficiencies, new therapies, new diagnostics, and new economies. Already, Al is having an impact on healthcare, and new prospects of far greater advances open up daily. This paper sets out how Al can bring new precision to care, with benefits for patients and for society as a whole. But it also sets out the conditions for realizing the potential: key issues are ensuring adequate access to data, an appropriate regulatory environment, action to sustain innovation in research institutes and industry big and small, promotion of take-up of innovation by the healthcare establishment, and resolution of a range of vital legal and ethical questions centred on safeguarding patients and their rights. For Eu-
\end{abstract}

rope to fulfil the conditions for success, it will have to find a new spirit of cooperation that can overcome the handicaps of the continent's fragmented technical and legal landscape. The start the European Union has made shows some ambition, but a clearer strategic vision and firmer plans for implementation will be needed. The European Alliance for Personalised Medicine (EAPM) has listed its own priorities: data, integrating innovation into care, building trust, developing skills and constructing policy frameworks that guarantee infrastructure, equitable access, and legal clarity.

(c) 2019 S. Karger AG, Basel

No one realised how drastically the world was about to change after steam power started its wholesale re-engineering of industry and transport some 200 years ago. In the same way, the implications of the work of Tesla or Edison were undreamt of as these 19th pioneers of electricity pushed back the frontiers of technology and ushered in a new age of comfort, convenience, and connectivity. And as the 21 st century gathers pace, the world is poised on the brink of another transformation, every bit as revolutionary: artificial intelligence (AI) is re-defining how civilisation organises itself. In everything, from space

\section{KARGER}

(C) 2019 S. Karger AG, Basel 
exploration to advancing life sciences, and from developing autonomous cars to tackling money laundering, $\mathrm{AI}$ is prising open the door to unprecedented new opportunities.

The health sector stands to be one of the major winners from the application of AI. Personalised and precision healthcare can become a reality rather than a concept, patient outcomes can be dramatically improved, healthcare professionals will acquire new power, and efficiencies can be driven up across the health sector [1].

AI goes much further than the innovations of steam or electricity. For the first time in the history of humanity, systems are emerging that are capable of a degree of autonomy: AI provides a technology capable not only of rapid and super-human analysis, but also of taking actions accordingly to achieve specific goals - unlike traditional rule-based software, which requires specific instructions to complete a task. The flexible mathematical models at the heart of AI exploit multiple algorithms to identify complex non-linear relationships within large datasets. At its simplest, AI is already, on a daily basis, translating languages, generating subtitles in videos, or blocking email spam. But its potential is limitless. Machine learning and deep learning, a type of AI, allows computers to "learn" without being explicitly programmed. In any given domain, it can help improve and automate decision making [2].

\section{What Al Can Do for Health}

AI in healthcare is already a reality. Devices based on AI are bringing new sophistication to image analysis. Deep learning algorithms are currently used in mammography for breast cancer detection, in CT for colon cancer diagnosis, in chest radiographs for the detection of pulmonary nodules, in MRI for brain tumour segmentation and for the diagnosis of neurologic disorders, such as Alzheimer's disease. Algorithms can help dermatologists make better diagnoses, for example detecting $95 \%$ of skin cancers by learning from large sets of medical images [3].

The use of AI in radiology has the immediate benefit of easing and accelerating workflows for standard reading of images, but it is also on course to do more, leading to a better and more precise understanding of disease. Predicting the course of cancer from imaging data could become a part of the clinical routine in the next few years. The increasing use of AI in radiology holds out the prospect of fundamentally changing the interpretation of medical images. It could also lead to a more exact under- standing of disease - for instance through the prognosis of disease progression based on hitherto unused imaging information. Image reconstructions from imaging data and radiological reporting are facilitated considerably by AI. Besides this, key values and parameters can be established more accurately and rapidly: lung nodules and tumour foci can be precisely measured, lung volumes automatically quantified, and calcium scoring speeded up [4].

When AI solutions remotely assess a patient's symptoms and deliver alerts to clinicians only when patient care is needed, it reduces unnecessary hospital visits. It can also lessen the burden on medical professionals. AI can save $20 \%$ of nurses' time [5] by averting unnecessary visits. The improvements will be felt at the level of healthcare systems, as well as by individual patients. Innovative solutions making use of digital technologies can provide support for reforms in the organisation of healthcare and long-term care systems. The European Commission speaks of how "artificial intelligence and supercomputing offer new opportunities to transform healthcare systems" [6].

\section{The Potential for Healthcare}

The goal should be to integrate AI into Europe's healthrelated operations, so as to improve clinical care, drive new therapies and treatments, and make healthcare systems more efficient. The implications for health are huge.

AI in healthcare is already a reality. Healthcare providers are starting to embed the technology into their workflows and the decision-making processes at all stages of healthcare, bringing improvements for patients, providers, payers, and other healthcare stakeholders, as well as society at large. The benefits range from new treatment opportunities - such as in detecting suspicious lesions that may have otherwise been overlooked - to back-office areas such as claims management and instrument maintenance programs [7].

With AI, patients will receive more rapid and accurate diagnoses than is possible with current systems, and AI will assist in decision-making, allowing for tailored treatment interventions with higher first-time success rates. Healthcare delivery can be improved, and adverse events reduced. Personalising care means more effective care, less waste of time and resources, greater patient satisfaction. There are significant cost savings to be derived from deploying AI, too - particularly in the better-targeted care it permits. It can even ease some of the growing strains in staff shortages across European healthcare centres [8]. 
The most exciting innovations are in frontline care, where patients will, over time, benefit most directly. In Denmark, AI is helping save lives by allowing emergency services to diagnose cardiac arrests or other conditions based on the sound of a caller's voice [9]. In Austria, AI is helping radiologists detect tumours more accurately by instantly comparing X-rays with a large amount of other medical data [6]. Large-scale analysis of patient-reported symptoms or biometrics can suggest potential diagnoses and next steps for patients.

AI allows monitoring of patients to promote improved outcomes. Patients and physicians have access to continuous assessment and support in areas such as compliance, through applications such as AiCure [10], a smartphone interface that ensures patients take their medications correctly, and a range of AI chatbots are designed to give real-time support and advice to patients receiving treatment - such as Sophia, Novo Nordisk's chatbot to help adherence by diabetes patients [11].

Research and innovation in diagnosis and treatment are also in line for massive advances as a result of combining new volumes of data with the processing capacity of supercomputers and the analytical assets of AI. AI opens up the way to a switch in discovery methods for drug development. For instance, a new cancer-fighting product now in clinical trials is derived from AI-based monitoring of trillions of data points from both cancerous and noncancerous cells. This is light years ahead of the traditional discovery method at the heart of classic drug discovery - a method with a less than $10 \%$ success rate. AI approaches are projected to halve the development cost of a single drug from USD 2.6 billion to 1.3 billion [12].

AI-based models are already helping clinical trial design and patient recruitment, and AI-based monitoring systems are being employed to boost study adherence and decrease dropout rates. "AI is not a magic bullet and is very much a work in progress, yet it holds much promise for the future of healthcare and drug development," says computer scientist Stefan Harrer in Trends in Pharmacological Sciences [13].

\section{Sharper Diagnosis}

AI solutions have been developed that work with multiple data types, including patient-reported symptoms, biometric and biosensor data, imaging, and biomarker data, right through the patient care pathway from initial triage to monitoring of ongoing treatment. Sensors can detect symptoms at early stages and predict a potential health incident ahead of time, permitting preventive measures [14].

AI: Power for Civilisation - and for Better Healthcare
AI deployment in diagnosis-related applications for oncology, neurology, and cardiovascular diseases is showing encouraging results. Research from the Catholic University of Leuven, Belgium, has shown that AI was twice as accurate as pulmonologists in interpreting the results of respiratory tests, correctly diagnosing the primary disease in $82 \%$ of 1,430 Belgian patients versus the doctors' $45 \%$ [15].

The NHS in England is setting up a national AI laboratory, alongside a GBP 250 million investment for AI within the health service, on the conviction that analysing large quantities of data will allow it to spot trends, promote transformation of the life sciences sector, and improve the health outcomes of patients. Leading UK-based biotech firms, such as Cambridge Cancer Genomics, GTN, DeepMatter, and Exscientia, are already operating in this innovative environment [16].

\section{More Personalised Care}

AI enables the optimization of clinical pathways for individual patients. In complex diseases, such as cancer, new challenges are emerging with new science, and AI offers a potential pathway to resolution. Where effective drug combinations are required for significant therapeutic impact, as is increasingly the case today, it becomes more difficult to design effective drug combinations and to choose the right drug combination for the right patient as the drugs become more specific to molecular targets. AI, with the new ability to efficiently analyse datasets that focus on the specific disease of interest, brings additional power to the design of approaches. "AI platforms can rationally design optimal drug combinations that are effective and based on real experimental data, rather than on mechanistic assumptions or predictive modelling," says the Commission [17].

Healthcare uses machine learning to predict which treatments would be of most benefit to individual patients, and applications can provide customized health advice based on a user's specific genetic make-up. The examples are numerous: a wearable vest can detect breast cancer at early stages; devices can remotely monitor wearers for heart irregularities and improve cardiac monitoring and rhythm management; a predictive analytics platform identifies people whose health is most likely to deteriorate, and implements change protocols to prevent catastrophic health events; UK's NHS has been interacting with Google DeepMind's Streams app, which helps alert clinicians to acute kidney injury in patients [18]. 


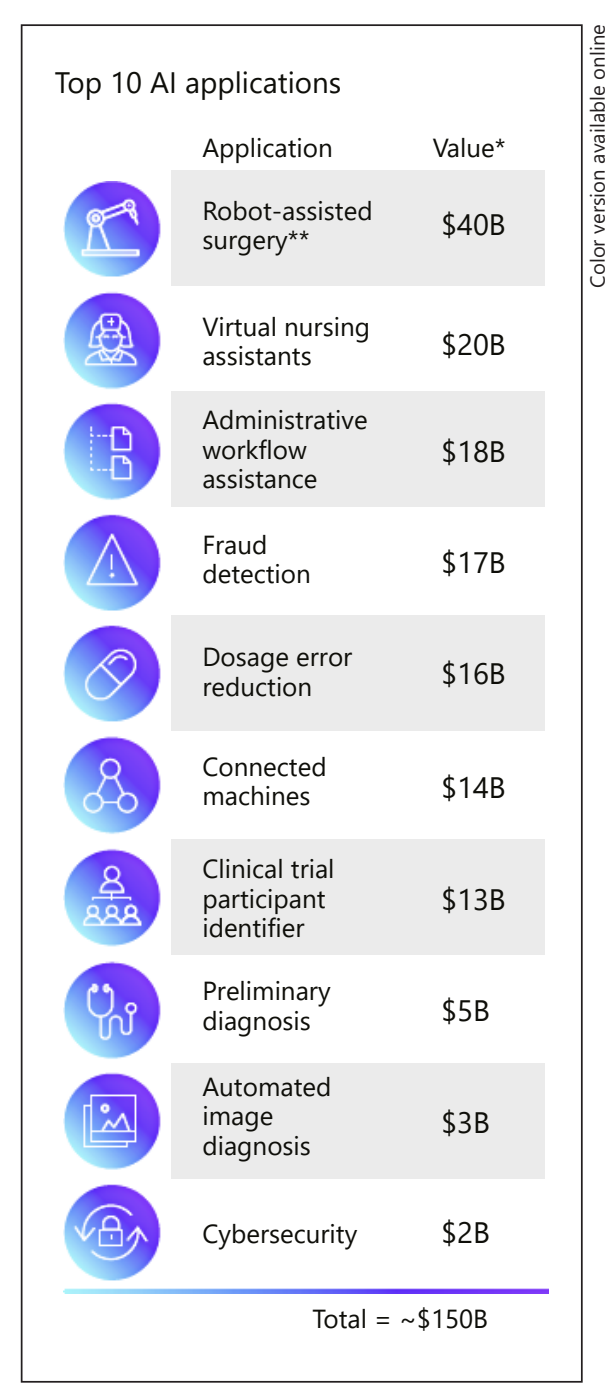

Fig. 1. Top 10 artificial intelligence (AI) applications. Source: Accenture analysis. * "Value" is the estimated potential annual benefit for each application by 2026 ; ** Orthopedic surgery specific.

Digital technologies can themselves enable citizens to access information about health risk factors and well-being measures, and they can help them engage in healthy lifestyle behaviour and disease prevention [17].

\section{Economic Benefits}

The benefits can be economic, too. As diseases are managed more effectively, payers will see cost savings associated with improved patient outcomes, such as a reduction in the number of complications. AI has the potential to yield cost savings of as much as EUR 90 billion over the next 10 years in obesity [19]. The savings would come from a combination of deployments: lower medical costs and reduced losses from low productivity and sick days; analys- ing clinical data in infants to predict future obesity and allow targeting of those at risk; studying the impact of an intervention among different populations, helping to expand or modify an approach; and improving understanding of obesity and risk factors related to genetics, metabolism, and environmental, lifestyle, and dietary habits [20].

It could save EUR 8 billion in costs of dementia over the next 10 years by improved accuracy of diagnosis and increased and earlier primary care diagnosis rates. Studies are also underway combining AI and MRI to measure blood perfusion in the brain as an aid to diagnosis of early forms of dementia [14].

And it could improve diagnosis and treatment of breast cancer through early detection, treatment decision-making, and reducing doctors' direct engagement in potentially repetitive tasks, saving up to EUR 74 billion over the next 10 years [21]. AI can analyse mammogram results 30 times faster than doctors with $99 \%$ accuracy. It could also help predict the degree of aggressiveness of detected cancers, permitting a more sophisticated response. And AI can help decide on a treatment plan likely to be successful among many different alternatives [22]. Its superior computation ability allows it to analyse data points as diverse as genetic data, DNA sequencing data, profiles, and electronica health records, as well as certain lifestyle data [23].

It could help reduce medical errors and adverse events, preventing more than 3.2 million days of hospitalization each year within the European Union (EU), with all the associated costs [14]. Accenture estimates that AI applications could save USD 150 billion in US healthcare costs by 2026 (Fig. 1). On the societal level, increases in overall levels of health and well-being will reduce costs of care.

\section{Efficiencies in Healthcare Management}

Healthcare providers and professionals can achieve improved efficiency of their workflows by having information readily and quickly available, reducing greatly the throughput and lead times.

AI can alleviate the significant and intensifying pressure of increasing staff shortages. UK's NHS, for example, has 45,000 clinical vacancies and a further 50,000 nonclinical open roles, and a similar lack of staff and capacity can be seen across Europe. Many of these positions are currently filled by temporary staff - a short-term fix that only serves to add further financial strain due to the higher costs associated with temporary employees. AI applications, such as those that conduct triage before patients arrive at a clinical facility, will give overstretched healthcare professionals greater leverage, allowing them to focus on interacting with patients on arrival [8]. 


\section{Realising the Promise}

Realising the promise of AI, robotics, and big data to improve clinical outcomes, reduce health system costs, and heighten the patient experience is the core challenge facing mature healthcare systems in the 21 st century. The full benefits of AI in healthcare depend on addressing challenges ranging from access to data and regulatory and technical issues to still unresolved legal and ethical questions [24].

\section{Data Front and Centre}

The goal should be to create EU-wide common data spaces that promote access to health data in a secure environment, with $100 \%$ of health data digitized within 5 years and maximum interoperability.

Data and data-related information are the building blocks of medical knowledge. As a consequence of new science and new techniques of data capture, the healthcare environment becomes increasingly data-rich, way beyond human capacity to take advantage of it all. But with AI, that treasure-house can be searched and interpreted in ways impossible using only traditional dataprocessing methods. AI will effectively enable us to make good use of big data in health. With AI, it is possible for systems to read unstructured data, identifying meaningful relationships in raw data, aggregating and displaying it, and offering analysis. By unlocking complex and varied datasets to develop new insights, AI can help physicians and researchers prevent disease, speed up recovery, and save lives. It can speed up genomics processing, aid in the development of advanced drugs and therapies, and contribute to more accurate personalized treatment. And it can be used to detect and correct waste, fraud, and abuse in healthcare spending [25].

It is now established European policy - as well as common sense - that data can transform healthcare, both at the level of the individual patient and at the level of healthcare system (see for instance the European Alliance for Personalised Medicine (EAPM)'s paper "Cooperating on data: the missing element in bringing real innovation to Europe's healthcare system" [26]). Patient information, medical records, diagnostic results, and clinical studies are just a few of the data sources available in healthcare. The digitization of patient records is an important contributor to this evolution. Big data can be retrieved from electronic archives - mobile phone applications, wearable technology, social media, environmental and lifestyle-related factors, socio-demographics, data from genomics, metabolomics, proteomics, radiomics, data from

AI: Power for Civilisation - and for Better Healthcare standardised electronic health records, or precision medicine platforms [27].

As the availability of data increases, so does the potential to provide better services and more effective therapies and treatments - which puts access to quality data front and centre in the era of AI and personalised care. But more is required than simply increasing the volume of data, if the aim is to create an effective data-based ecosystem allowing for progress in understanding and preventing diseases, and for improving personalised medical research, diagnosis, and treatment. What is needed is a clear view of what kind of data is effectively in store, what format it is in, and how reusable it is. The Commission observes, in its comments on exploiting genomic data: "A major challenge faced by healthcare authorities, practitioners and scientists is being able to bring this wealth of data together and interpret a genomic finding in the context of the individual patient. There is a need to share data, deriving the knowledge gained from data at a population level to sort through the 'noise' of variation in a person's genome to identify the 'signal', an individual change or pattern that may be unique to an individual." This will depend on investments in interoperability, data provenance, and curation. It needs actions to strengthen interoperability across multiple data sources and allow patients easy and secure access to their health data - something that Member States of the EU could put into effect by launching health data plans, and by going deeper and wider on health data digitization [6].

The quality of conclusions on the aetiology of diseases follows a law of large numbers. Cross-sectional cohort studies of 30,000-50,000 or more cases are required to separate the signal from noise and to detect genomic regions associated with a given trait in which diseaserelated genes or susceptibility factors are located. Even some of the largest national programmes to aggregate -omics data, such as the UK Government-funded 100,000 genomes project or France's Médecine Génomique 2025 which will establish capabilities for sequencing over 200,000 genomes a year, will have a limited number of individuals that share the same genetic mutation and indeed often relatively few patients with a specific condition, particularly for rare disorders [28]. Similarly, as the Commission's staff working document points out: "Personalised treatments for complex diseases like cancer require the ability to process very large amounts of data, while applying methods of interpreting data and extracting new knowledge such as computer modelling, machine learning or artificial intelligence" [6]. 
In the staff working document, the Commission endorses the view that the use of digital tools "allows the capture, management and processing of large volumes of diverse data generated from multiple sources to create new knowledge (and) enables new approaches to personalised medicine, accelerating scientific progress, early diagnosis and prevention of diseases and more effective treatments." The same digital tools "can assist in addressing shortages in health and care staff in rural areas and certain specialties. They can also connect the various actors across the health and social care sectors, thus ensuring effective sharing of data and collaboration, in more effective care models." In addition, "the analysis of digital health data and patient-reported data can lead to improved procedures, reduce inefficiencies, support outcome-oriented healthcare, promote the evidence-based assessment of innovative health technologies, as well as improve emergency preparedness and response to epidemics" [6].

The Commission goes on to point out that EU research projects - for instances COMPARE [29] and EVIDENT [30] - provide insight on how supercomputing capacity and AI, bringing together large datasets from different sources and disciplines, can improve the understanding of epidemics, and accelerate EU-wide identification of infectious threats. "Advanced IT technologies can also support the development of new risk models to predict the possibility and trajectory of potential future outbreaks, by facilitating the integration and comparative analysis of inputs from various data sources, e.g. from human and animal health data, vaccination coverage, vector surveillance, laboratory data, environmental data, population density, travel, etc..." [6].

The Lancet recently observed in an editorial: "From genomic and digitised demographic databases to individual health tracking recorded on smart watches to local pollution and weather monitoring data, the digital revolution is providing more and better data on the determinants of disease. The ability of artificial intelligence and machine learning algorithms to analyse these multiple and rich data types at a scale not previously possible could bring a step change in public health and epidemiology. Artificial intelligence could offer unprecedented accuracy and precision in predicting disease spread or the vulnerability of certain groups and to determine the most effective targets for interventions or the populations with the greatest need" [31].

Many AI technologies use data to improve their performance. Machine learning works by identifying patterns in available data and then applying the knowledge to new data. The larger a dataset, the better even subtle relations in the data can be discovered. When it comes to using AI, data-rich environments also provide for more opportunities. This is because data are the way the algorithm learns about and interacts with its environment. For example, if all machines and processes in a factory continuously produce data, it is likely that further automation and optimisation can be achieved with the help of AI. This is a sophisticated evolution that is not possible in an analogue setting, such as in a paper-based operation. Once trained, algorithms can correctly classify objects that they have never seen, in more and more cases with accuracies that exceed those of humans [32].

Useful stand-alone AI algorithms are already in clinical use for medical image interpretation, but the broader implementation of AI in daily healthcare will require versatile platforms that offer streamlined support to procedures and decision-making, allowing easy integration of the AI applications necessary to perform complex tasks into existing workflows and IT infrastructure [33].

\section{The Right Environment}

The goal should be to commit resources to development and adoption of $\mathrm{AI}$ and promoting its use, through testing innovative AI solutions in healthcare settings. As with any new technology, it is important that the environment should ensure adequate protection of the public interest as well as promoting valuable innovation. The context should be protective of the citizen, but it should also allow for experimentation and should support disruptive innovation across the EU.

For AI, there are several aspects that must be considered. By working together, the EU can maximise the benefits of AI in healthcare, ensuring that it delivers for patients, for healthcare, for society, and for the innovative strength of Europe. The objective is to maximise the benefits of AI for all Europeans by fostering the development of trusted AI that corresponds to European ethical values and citizens' aspirations, and to ensure cooperation among industry and academia in collaborative R\&D and innovation [6].

Software and algorithms that approximate human cognition can support decisions in a way that has previously been the domain only of humans. But rather than replacing the human component of healthcare, the true art lies in deploying AI as an essential tool in healthcare - a companion for physicians to help them improve patient outcomes. As AI develops and is implemented on a wider scale, it promises to allow health professionals to focus on the more challenging tasks that $\mathrm{AI}$ is unable to 
replicate [8]. From the outset, however, pilot schemes and partnerships should start by defining clinical and health economic benefits upfront, then track progress. Demonstrating the benefits is critical to managers and payers, so their needs should be factored in explicitly.

Core datasets need to be identified and secure access provided. Locating credible and curated data will be essential - and so, too, will be compliance with data protection and privacy requirements. The General Data Protection Regulation (GDPR) recognises data concerning health as a special category of data, and it provides the regulatory framework, including safeguards, for the effective protection of such data. Innovative technologies, such as AI, require robust data protection safeguards to maintain the trust and confidence of citizens in the protection of their data, and AI providers should design such safeguards into their technology platforms and ensure they are actively communicated to all stakeholders [34].

Tools will need to be co-developed with clinician leaders to avoid regulatory pitfalls in the future as the regulatory environment for AI deployment in healthcare evolves. Similar joint work will be needed by regulators and payers to develop liability management frameworks - to be able to respond to situations such as if a patient is incorrectly triaged by an AI system, so as to provide guidance on who is at fault [35].

Practitioner uptake will be crucial in promoting AI adoption, and that will require support. Healthcare AI companies will need to form partnerships with care organisations and run pilots with universities and hospitals, so that healthcare practitioners get early access to their applications and are able to influence their development - and in turn lower the barriers to implementation. And training and education on $\mathrm{AI}$ in healthcare must be developed for patients, medical professionals, healthcare organisations, policymakers, and governments. AI technologies and applications will need to be trustworthy and "ethical and secure by design."

\section{A Regulatory Framework Built on Trust and Understanding}

The goal should be to build trust by promoting the engagement of all healthcare stakeholders to understand the technology, and training and education for patients, medical professionals, healthcare organisations, policymakers, and governments.

In the context of AI, a common question is whether the existing regulatory framework is fit for the purpose. The art of good regulation is finding the right balance. The Commission itself says that systems for approving new

AI: Power for Civilisation - and for Better Healthcare medical devices must provide pathways to market for important innovations, while also ensuring that patients are adequately protected.

The EU features a robust and balanced regulatory framework concerning liability and safety of all new products and technology (including via the Product Liability and Machinery Directives). On top of that, healthcare is a specifically regulated sector. Healthcare AI technologies with a medical purpose are already required to ensure a high level of safety and performance and demonstrate compliance with general safety and performance requirements defined by the Medical Device Regulation, providing for a high level of trust. The majority of the AIbased medical devices on the market today focus on image recognition and have had to demonstrate safety and clinical and analytical validity to be certified, including by comparing the algorithm's results over a large number of samples (such as lung scans) against those of human specialists (radiologists, in this case) [36].

It is important to recognise that the application of AI to a patient-level decision will be impacted by the quality and completeness of the available health data to inform its reasoning. Since electronic health records inevitably contain data of variable quality and completeness, it is important that AI will always "fail safe" and indicate transparently to the user if there is insufficient data to arrive at a conclusion or to make a recommendation, rather than provide an incorrect answer. This requires robust safety testing.

Standards and definitions need to be endorsed on the European level. There is a need to clarify how existing legislative frameworks can be made more inclusive so that all forms and applications of AI in healthcare benefit from the same legal clarity and certainty. Many legitimate questions still need to be explored. How can society derive the most value from health data without compromising patients' privacy? Where does liability ultimately fall? Is the healthcare workforce ready and trained for these revolutionary changes? How to promote access in a nondiscriminatory manner?

\section{Integrating Ethics}

The practice of healthcare has strong ethical roots. This must not change with AI. Addressing accountability, privacy, transparency, equity, and safety is necessary to ensure trust in healthcare AI. European initiatives to build voluntary Ethics Guidelines for AI in healthcare are welcome, as these would help set world standards for AI and simultaneously give the reassurance needed to society to trust these technologies as well as for industry to 
invest further. The High-Level Expert Group on AI (https://ec.europa.eu/digital-single-market/en/high-level-expert-group-artificial-intelligence) has put forward Ethics Guidelines for trustworthy AI which may provide a base to address the specificities of the healthcare sector [37].

\section{Ensuring Accountability}

Organizations should embrace risk-based accountability approaches, putting in place technical or organizational measures, such as product development lifecycles and ethics review boards, to minimize risks in AI.

AI is still, in institutional terms, in its infancy, and there is inevitably some lack of clarity about its usage. The nature of AI-based solutions, in which advances depend on a degree of AI self-learning and reason, prompt questions about the safety and effectiveness of decisions or recommendations. For AI in general, this question of accountability presents a particular challenge. The issue is already being demonstrated in discussions on other AI applications, such as over who is responsible when a driverless vehicle under the control of AI makes a faulty decision. This scenario does not apply directly in the healthcare environment, where the AI's role is merely a support tool. But there will have to be considerations at some point, if AI starts making autonomous decisions about diagnoses and treatments. The question will arise as to whether the AI developer can be held accountable for its decisions. So, too, will questions about the role of healthcare professionals and, more broadly, the impact for the patient.

Central to the current approach to AI in the healthcare sphere is a distinction between AI's use in areas with potential patient impact and its use in wider and less patientspecific areas, such as research, or healthcare management improvement. AI-based systems come in many shapes and sizes, depending on their purpose, and while general regulations on safety and liability will apply in all cases, specific rules will apply to those systems that have a direct implication in patient care and treatment. Systems can be purely software-based, acting in the virtual world (e.g., voice assistants, image analysis software, search engines, or speech and face recognition systems) or they can be embedded in hardware devices - advanced robots, drones, or Internet of Things applications [38].

So not all uses of AI-based solutions in healthcare raise the same concerns. For example, solutions aiming at streamlining hospital administrative tasks would attract different concerns than, say, a solution that suggests a certain disease diagnostic or recommends a course of thera- py. Non-medical uses of AI in the healthcare sector range from supporting clinical research and drug development to operations and administration. AI application in nonmedical uses demands the same quality characteristics (e.g., functionality, reliability, usability, and efficiency) as any other software product, but not the same specific rules related to patients' health in entirely medical applications. Programs that analyse large amounts of data to develop knowledge about a disease or condition, rather than to decide on treatment options for an individual patient, may not necessarily be considered as having a medical purpose, and hence as being a medical device. It is the basic distinction between those research programs that enhance medical knowledge from those that promote changes in healthcare [14].

In terms of healthcare and its direct engagement with patients, it is more apt, perhaps, to speak of "augmented intelligence" rather than "artificial intelligence," since the technology "augments" the capability of an individual, rather than becoming a replacement. The use of AI in healthcare inevitably provokes reflection about the role of healthcare professionals and its impact on patients. It should be designed and used to improve patient care by augmenting healthcare providers' capabilities.

The distinction between medical and non-medical use applies directly to this discussion. Many of today's applications of AI in the medical sphere address clinical decision support. These applications support (or augment) the healthcare professionals' abilities in delivering highquality care, for example, by pinpointing abnormal looking cells on an image of a patient's tissue sample, which could have escaped detection by a specialist doctor. As AI in healthcare matures, it is expected that humans will increasingly partner with AI solutions, and the discussions will become more complex. But it is important that AIbased solutions with a medical purpose should always be intermediated by licensed medical professionals. This does not, of course, exclude the possibility that individuals will safely be able to benefit from AI on their own, without limiting the use of AI to medical professionals or to only under their supervision [39].

Currently, AI-based medical devices are "locked," i.e., the AI software does not autonomously learn after it is certified for market use. However, the current regulatory framework for medical devices is not adequate to ensure the safety and efficacy of adaptive, continuously learning AI systems. While these may be some time out in the future, inevitably this would require a different regulatory paradigm. In the USA, the FDA initiated a reflection process regarding the regulatory framework for modifica- 
tions to AI-/machine learning-based software as a medical device. It aims to address the iterative improvement capabilities of $\mathrm{AI} /$ machine learning software, while assuring that patient safety is maintained [40].

It will also be important to ensure that there is adequate professional and public understanding of AI and its role. As AI matures, its acceptance by the healthcare sector will continue to emphasize safety and effectiveness, and this will have to take transparency into account, so that users have enough understanding of the technology, its uses, and limitations.

To be able to assess the outcome of an AI-based decision, concerns could remain on how the AI interaction between algorithm and data actually works and how to ensure that the results are explainable to a human. This will be all the more important when it becomes very difficult or impossible for a person to comprehend all the data and dependencies involved in the AI process. In these circumstances, explainability (or interpretability) of an AI decision becomes very important. To a large extent, explainability depends upon the AI technique utilised. The way some techniques operate may make it more (e.g., deep learning) or less difficult to "reverseengineer" an AI decision, i.e., to understand how the algorithm reached a certain result. The industry fully recognises the need to provide meaningful information and facilitate the interpretation of algorithmic decisions of AI in healthcare. The level of AI explainability should be commensurate to the intended use and risk for the patient and understood as the provision of meaningful information to the user. It should not be about sharing source code or training data. In addition, study results demonstrating the safety, clinical and analytical validity of the solution should be the main evidentiary factors [41]. Industry and academia researchers are already developing techniques giving more information and context behind AI-driven decisions. Principles and guidance are being produced to assist developers in making systems auditable from the start.

Organizations should embrace risk-based accountability approaches, putting in place technical or organisational measures, such as product development lifecycles and ethics review boards, to minimize risks in AI. The industry has developed product development lifecycles, which include impact assessments and balancing tests to measure privacy and security risks of AI-based technologies. A growing number is including ethical impact assessments. Future technologies are increasingly discussed within companies' ethics councils or ethical review boards [42].

AI: Power for Civilisation - and for Better Healthcare

\section{Taking Care of Patients and Their Rights}

The goal should be to guard against any unintended consequences of the use of AI, through appropriate policy frameworks that preserve privacy while permitting innovation, and that address data bias and promote equitable access.

Many patients seem willing to share their health data: according to a survey from the European Commission, $80 \%$ of Europeans would agree to share their health data if privacy and security are ensured. But the extent to which it does not yet deliver for clinical and research purposes is well documented. For example, the European Commission remarked that "it is widely shared among the stakeholders that access to varied data-sets located across different Member States remains difficult or inexistent, the data is subject to different taxonomies and standards and therefore scientific research invariably builds on relatively limited population cohorts." And an analysis of data-sharing guidelines issued by multiple organisations "observed substantial fragmentation in the policy landscape across specific organizations and data types" and that "data are not being shared to a degree that can trigger the expected data-driven revolution in precision medicine" [6].

Patients have indicated that they are ready to integrate AI into their healthcare experiences. A recent survey for Pega systems showed that $42 \%$ of patient respondents were comfortable with their doctors using AI to make healthcare decisions, and $29 \%$ with their payers doing so. But a recent survey of 29,000 EU citizens suggests that drug firms will certainly have to be more persuasive if they want to collect people's DNA details. Only 4 in 10 respondents said they agree with the statement "I'm willing to share my genetic information with pharmaceutical companies." Almost half explicitly disagreed, with about 1 in 4 saying they "strongly disagree." When it comes to sharing genetic info with academic researchers, Europeans are more open, with $58 \%$ in favour. Some $76 \%$ said they are willing to share data with family members, and $73 \%$ are keen to know "as much as possible" about their own DNA [43].

Focus group discussions showed that people want to be able to make autonomous decisions - but in order to obtain truly informed consent, sophisticated explanations will be needed about genomic data, what it means and how it will be used. That in turn will require new training for health professionals - indeed, new types of health professionals like genetic counsellors may need to be recognized.

The reasons for a suboptimal use of health data are multiple. A report focusing on oncology data sources in 
Europe highlights challenges related to limited health data digitization, quality, format and accessibility; lack of sustained political focus on health data; heterogeneous and time-consuming patient consent frameworks; limited technical interoperability, and concerns about data sharing by healthcare providers themselves [44].

In addition, data must be Findable, Accessible, Interoperable, and Reusable. Following FAIR data principles as a basis for the governance of health data means that health data is stored according to widely accepted standards to facilitate its search; that technical and organizational measures are in place to securely provide access to the data; that the quality of the data follows widely agreed metrics and standard formats, and that attribution is assured so that incentives and rewards for data sharing are in place [45]. The large-scale secondary use of health data for research and AI should be explored through pilots, and a healthcare-specific AI ethical framework should be agreed on.

\section{Respecting Privacy}

We must focus on regulatory and technological options to maximize the value of health data without compromising patients' privacy. Ensuring appropriate rules and practices in relation to privacy for the individual is another dimension requiring urgent attention. The growing use of AI in healthcare - based on new large volumes of data - will intensify discussions of privacy and security of patient's health data. As the European eco-system for health data develops, so will data collection, collaboration amongst stakeholders, and opportunities for data processing. In this context, concerns about privacy and data protection will equally increase.

In Europe, processing of data concerning health is regulated by the GDPR. But while the GDPR is an EU-wide data protection law, it does not completely harmonize data protection rules across the EU. In particular, individual EU Member States are allowed to adopt national rules that "supplement" the GDPR, for example, in relation to the processing of health or genetic data. It is, thus, very important that Member States and the European Commission minimize, or even better eliminate, regulatory divergences regarding data concerning health.

Codes of conduct are a means to ensure the proper application of the GDPR, reinforcing trust by demonstrating compliance to the public and regulators. Within the context of healthcare research, for example, codes of conduct would contribute to network effects because their increased adoption by stakeholders would lower the barriers to enter in large-scale collaborative research initiatives [46].
An example that other Member States are invited to consider is Finland's Act on the Secondary Use of Health and Social Data. The Act addresses the secondary use of health and social data and establishes a "one-stop shop" - the Data Permit Authority - to grant access permits for health and social data. It does this in a centralised manner, when a data enquiry requires collection from several data repositories managed by different organisations. An operating environment with robust cyber security controls will be created in which the data disclosed can be processed in accordance with the permit, although processing in other environments is also foreseen [47].

\section{Safeguarding Individuals and Ensuring Equitable \\ Treatment}

Access needs to be promoted in a fair, transparent, and non-discriminatory way. Equally important is ensuring that advances resulting from AI are widely accessible to entire populations and equitable in their approach to protecting individuals. It must be remembered that "digital technologies are not ends in themselves," but tools to promote health - as WHO Director-General Dr. Tedros noted on April 17, 2019, at the launch of the first WHO guideline on digital health [48].

As AI solutions and platforms become increasingly common and affordable and improve continuously, it is essential to ensure that patients will have easy access to this type of specialised and personalised healthcare delivery. As the use of AI in healthcare expands, its benefits should remain accessible to all, while the workforce and patients should be active in informing this transformation.

One of the challenges is to ensure that the algorithms on which $\mathrm{AI}$ in general relies do not err in mirroring any unjustifiable human biases and do not reflect widely shared values. Algorithms based on limited or flawed data could end up integrating prejudices of a racial, cultural, gender, or social nature. AI applications introduced in some fields have already been shown to make problematic decisions that reflect biases inherent in the data used to train them. Recently, for instance, a program designed to aid judges in sentencing by predicting an offender's risk of recidivism have shown a propensity for discrimination. It is, therefore, appropriate that the developers of AI are transparent about the populations of patients and health scenarios the AI has learned from, and for which it may, therefore, be most confidently used. Adopting the same AI with other populations and health situations needs to be undertaken with caution and probably additional validation. 
In healthcare, it will be necessary to guard against errors arising from inappropriate AI models and data. Since healthcare delivery already varies by ethnicity, it is possible that some ethnical biases could inadvertently be built into medical algorithms. At its simplest, an algorithm designed to predict outcomes from genetic findings may be biased if there are no genetic studies in certain populations. At its worst, private-sector designers who create AI algorithms for clinical use could be tempted to programme systems so as to guide users towards clinical actions that would give priority to profits rather than patient care, by recommending particular drugs, tests, or medical devices in which the system developers hold a stake [49].

For AI to be beneficial for healthcare, there needs to be confidence that the manufacturers of AI systems make objective decisions and are not skewed by bias hidden in the data that were used to train the systems. AI models will never be completely free of bias, as bias permeates our world and societies. But much can be done to minimize the risks and constantly improve models. Requiring that the dataset used for training, tuning, and validation is representative of the population that is targeted by the AI system is an obvious approach. And there should be clear articulation of boundary conditions under which the AI model is intended to be used, covering issues such as the range of inputs or the target population. Quality management systems would track data and model provenance and version history. A "gold standard dataset" can be used as a reliable metric to determine model performance and detect errors before the model is updated [50].

A good application of AI may be powerful, helpful, and valuable. On the contrary, a bad or unethical use of this cutting-edge technology may be dangerous, and industry, patients, physicians, and regulatory authorities must work together to prevent this. The development of AI in Europe should be based on "ethics by design," which means factoring in ethical and legal principles based on the GDPR, compliance with competition law, and safeguards against data bias from the outset. "An appropriate and predictable, ethical and regulatory framework that relies on effective safeguards for the protection of fundamental rights and freedoms is vital for citizens to trust AI and for companies, in need of investment security, to take up new business opportunities," argues the Commission [51].

\section{Getting Regulation Right}

The EU must ensure that AI is developed and applied in an appropriate framework which promotes innovation and respects the EU's values and fundamental rights as well as ethical principles such as accountability and transparency. But overall, the EU needs to position itself so as to play a leading role in the adoption of standards, so that they can influence the emergence of standards at world level. The EU is well placed to lead this debate on the global stage [52].

For all these outstanding questions, on complex matters of liability, negligence and fault, or the attribution of risk and accountability, further analysis should be conducted in an informed and evidence-based manner. Account should be taken of the practical experience of the Product Liability Directive and the Machine Directive, which have worked and continue to function well [51].

Whatever form regulation of AI takes, it is important that policy frameworks for $\mathrm{AI}$ in healthcare are risk-based and take full account of existing legislation, standards, and ethical principles. Risks should be identified in the first place to assess whether existing legislation is adequate. Regulation of clinical AI solutions should be based on their intended use and the risk it presents to patients. We invite the EU to establish a dedicated forum or leverage existing initiatives, such as the medical devices coordination group (MDCG) of the European Commission, to further address the regulatory aspects of $\mathrm{AI}$ in healthcare in Europe and to promote communication and collaboration between data scientists, medtech experts, and academia. Agile risk-based certification frameworks will be needed for AI-based software medical devices.

It is also crucial to include consideration of the appropriate business model as the regulatory framework evolves. Current reimbursement systems do not provide premium reimbursements where AI solutions are being applied, additionally to the existing reimbursement.

\section{The Wider the Cooperation, the Better the Outlook}

Successful adoption of AI - in general and for healthcare in particular - requires multi-stakeholder commitment to address both the opportunities and the challenges. Europe still has to maximise its potential in this domain. European investment lags other major regions in the world, and it is frequently lacking in coherency. Europe also suffers from skills shortages in relevant domains [41].

\section{Europe's Chances and Risks}

On the brink of this radical transformation in the technology and effectiveness of healthcare, Europe is in many respects well-placed, with its vigorous life-science sector, 
skilled workforce, and highly developed healthcare services. But Europe is not set up for the AI revolution. Fragmented national datasets make it harder to compete. Without significant investment and government-driven co-ordination in Europe, there is a risk that Asia and North America will take the lead in AI innovation. For Europe to have a chance at competing with the USA and China on AI, we needs to harmonize our approaches, attract talented programmers back to Europe, and put in place a framework based on European values.

Big investments are being made in AI. AI deal activity across healthcare and life sciences has surpassed all other industries since 2013. Globally, healthcare AI companies raised USD 4.3 billion across more than 500 equity deals in this period. Funding levels and deal volume have grown significantly since 2013, with 2018 on track to significantly exceed 2017 levels. But Europe is not in the lead. Global investment and financing in AI are clearly led by China from 2013 to the first quarter of 2018 , with $60 \%$ of the total. And in 2017, China confirmed its dominance by accounting for $70 \%$ of the USD 39.5 billion invested in AI worldwide that year [8].

Investment levels for $\mathrm{AI}$ in the EU are low and fragmented, relative to other parts of the world such as the USA and China. The government of the USA presented an AI strategy and already invested around EUR 970 million in unclassified AI research in 2016. With its "Next Generation Artificial Intelligence Development Plan," China is targeting global leadership by 2030 and is making massive investments. Other countries, such as Japan and Canada, have also adopted AI strategies. And in the USA and in China, large companies are significantly investing in AI and are exploiting large amounts of data. Overall, Europe is behind in private investments in AI which totalled around EUR 2.4-3.2 billion in 2016, compared with EUR 6.5-9.7 billion in Asia and EUR 12.118.6 billion in North America [51] (Fig. 2, 3).

\section{Overcoming Fragmentation}

Incentives for cooperation will be needed between care providers and the AI sector. Ensuring that the promise of AI in healthcare can become a reality will not be straightforward. Europe needs to engage in a broad dialogue to overcome any barriers to wide adoption, and this will have to involve healthcare providers and physicians, patients, payers, industry, policy makers, and other relevant stakeholders. The European Commission itself insists that it is, therefore, crucial that the EU continues its work to create an environment that stimulates investments and uses public funding to leverage private investments.
Europe must also tackle human resources challenges because it suffers from a significant and persistent information and communication technology skills gap. Demand for skills in emerging areas such as AI are particularly acute, and the problem is growing as the offer lags behind the market. Almost all Member States are facing shortages of information and communication technology professionals, including in the area of AI [53].

It is equally important to foster the broadest possible uptake of AI in the economy, in particular, by start-ups and small- and medium-sized enterprises (SMEs). By raising public awareness and sharing the latest scientific advances and tried and tested state-of-the-art technologies developed in Europe, we can ensure that every company, small or large, high-tech or not, as well as the public sector, can grasp these digital opportunities [51].

Only if both the Commission and Member States direct their investments and energies in the same direction and leverage significant private investments will Europe as a whole have an impact and establish its strategic autonomy in AI. If Member States are to maximise investments, pool important resources, such as data, and provide a seamless regulatory environment, they must put in place national AI strategies and relevant support measures.

\section{AI and SMEs}

In fostering the broadest possible uptake of $\mathrm{AI}$ in the economy, the role of start-ups and SMEs is pivotal, both in terms of its scale and its vitality. More than 22 million SMEs operating employed over 90 million people in the EU in 2015, in a range of sectors including healthcare [54]. These enterprises, creating over $85 \%$ of new jobs and around two-thirds of the total private sector employment [55], are also significantly involved in bringing forward AI projects. Recently, we have described how to master the challenges and opportunities of optimising the SME potential in modern healthcare systems and made a policy recommendation [56].

The European Commission has clearly identified promoting the growth and activity of the SME sector as crucial elements in its strategic objectives, in its Communications "Think Small First. A Small Business Act for Europe" [57] and "Small Business, Big World - a new partnership to help SMEs seize global opportunities" [58]. Our policy recommendation provides a summary of the recommendations, based on our engagement with national and EU policy-makers, politicians, and policy experts [59].

AI is embedded, implicitly or explicitly, in the following 5 areas identified as key to facilitating SMEs as drivers of health innovation: 


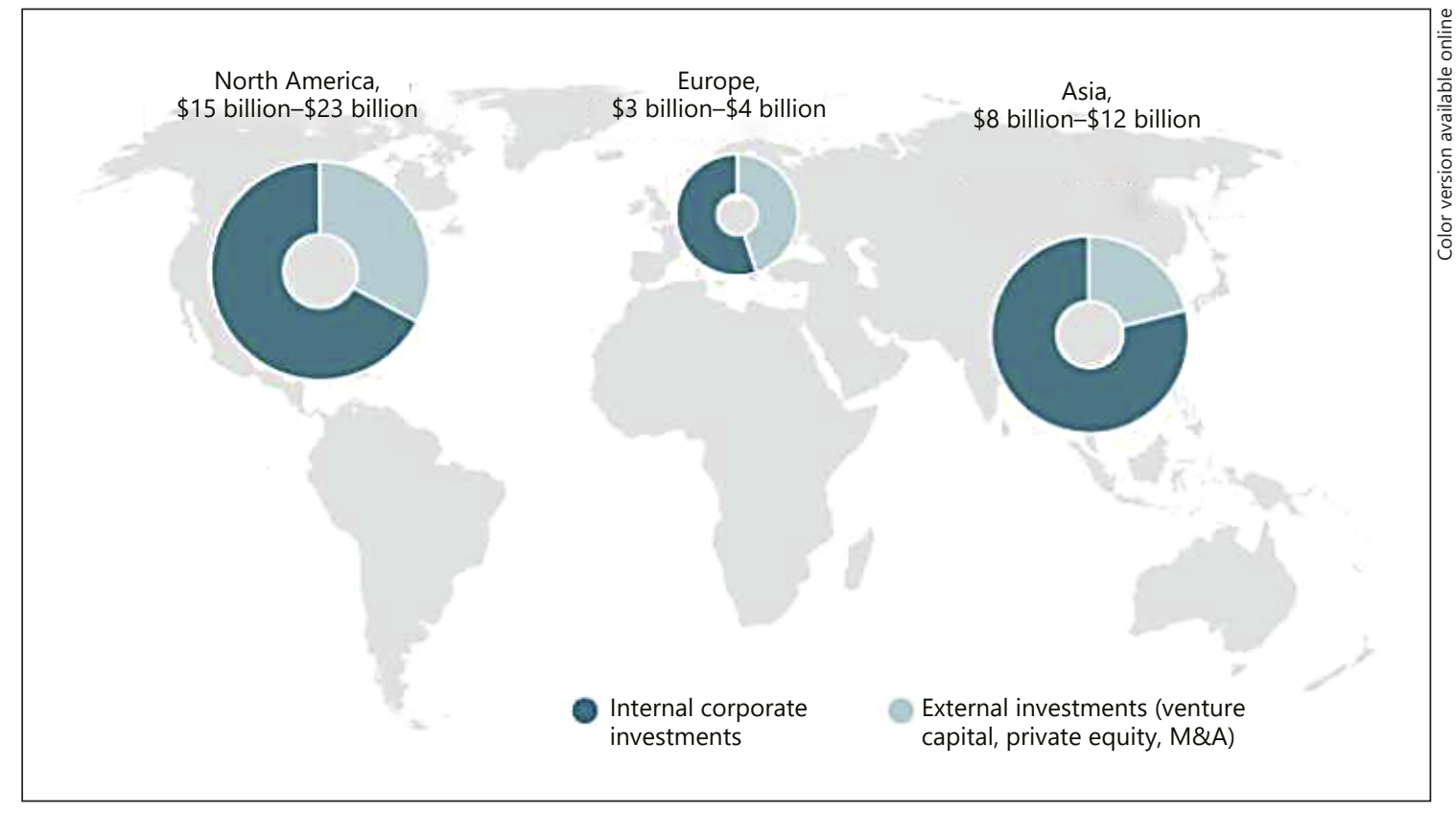

Fig. 2. Artificial intelligence investment, a comparison from 2016.

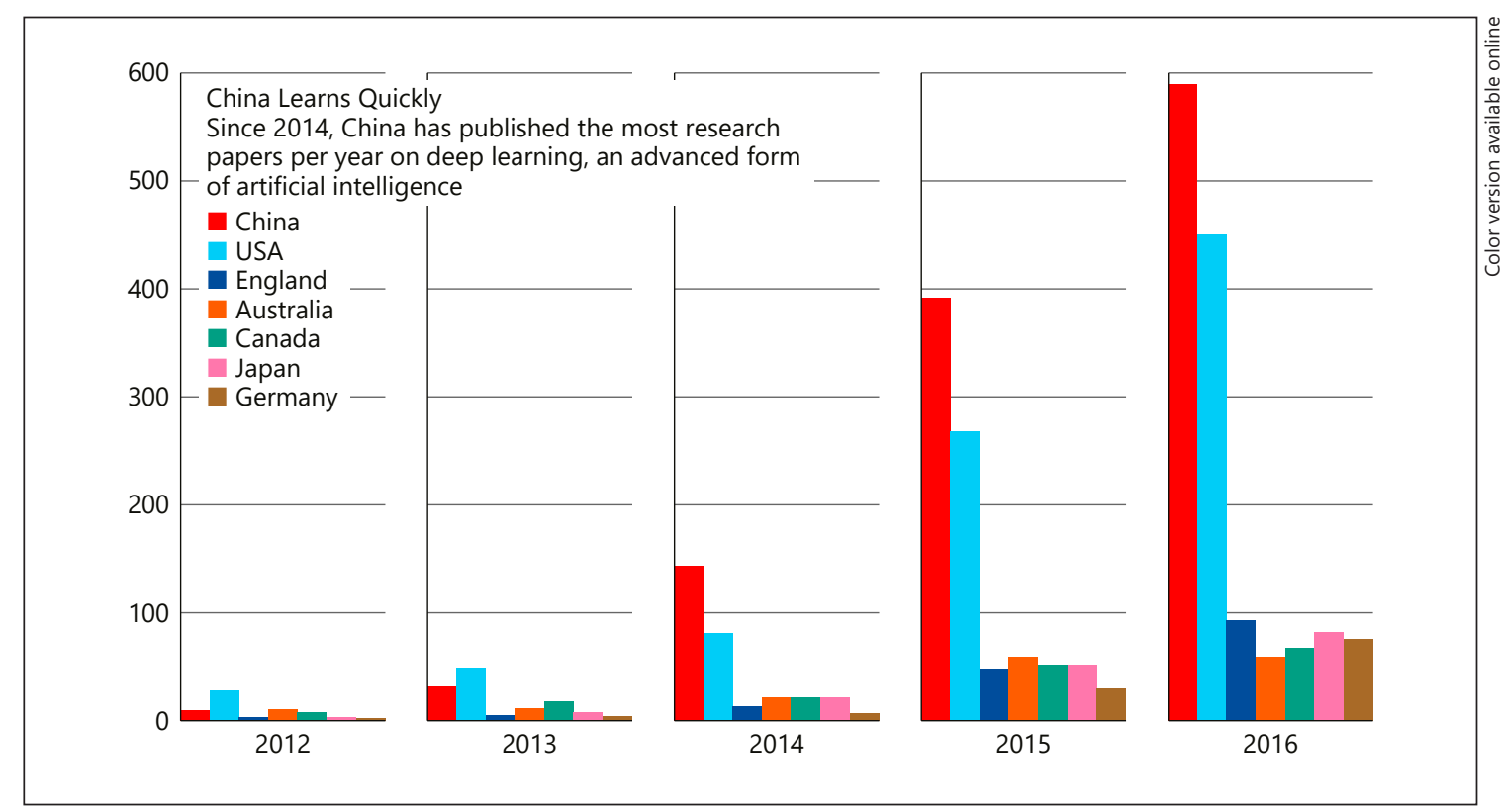

Fig. 3. Artificial intelligence R\&D papers, a comparison.

1. Frameworks: these are essential to the progress of new technologies and applications, including regulatory frameworks to fulfil new EU regulations.

2. Financing: recommendations include allocating a greater percentage of Horizon Europe to supporting
SME innovation in the field on feasibility assessment, innovation development, and demonstration purposes, and to define clear market access pathways to ensure further development and marketability of innovations 
3. Data: coordination between authorities on the secure exchange of data is needed to advance research and to overcome the technical barriers and costs that divergent standards present to SMEs' access to markets.

4. Market Access: the many opportunities for intra-EU trade for SMEs would be amplified with the creation of a stable regulatory framework to foster free movement of healthcare and AI products, which would promote their adoption in the technology and healthcare setting.

5. Access to Information: the numerous EU portals - providing access to innovation markets including advice on all aspects related to EU-funded research projects, such as first orientation, - should go further than their current offerings of strategy development, proposal preparation, implementation, exploitation, and promotion. They should also promote business network opportunities across borders and sectoral boundaries within and beyond Europe.

\section{Discussion}

\section{Europe Is on Its Way}

Things are starting to move in a more effective direction in Europe. In terms of technology, the EU is developing strong approaches in high-performance computing, data analytics, and AI, which can help design and test new healthcare products, provide faster diagnosis, and better treatments. And there are significant moves in terms of data acquisition and sharing.

Following commitments made by 19 Member States towards building a research cohort of at least 1 million sequenced genomes accessible in the $\mathrm{EU}$ by 2022, the Commission will support an initiative on linking genomics repositories. The Commission will also support building rare disease registries, in a way that takes full account of organisational, regulatory, security, ethical and technical compliance, and interoperability. This will allow AI technologies to be studied, developed, and trialled with a view to identifying new knowledge, support clinical research, and decision making [60].

In 2020, the Commission will support - in coordination with Member States - the development of a common database of health images initially dedicated to the most common forms of cancer (anonymised and based on data donorship by patients). Here, too, the work will comply with all necessary organisational, regulatory, security, ethical, and technical requirements. It will be combined with relevant AI tools to improve diagnosis, treatment, and follow-up. Overall, the initial EU contribution to these initiatives will be around EUR 35 million from the Horizon 2020 programme. Member States are encouraged to match this amount [32].

The solutions to Europe's skills shortages are going to be complex and long-term, but a start is being made on EU thinking in this area - although responsibility for education and training, including of teachers and trainers themselves, lies with Member States. According to the European Commission, the first challenge is to prepare society as a whole, which means helping all Europeans to develop basic digital skills, as well as skills which are complementary to and cannot be replaced by any machine such as critical thinking, creativity, or management. The EU needs to focus efforts to help workers in jobs which are likely to be the most transformed or to disappear due to automation, robotics, and AI. This is also about ensuring access for all citizens, including workers and the selfemployed, to social protection, in line with the European Pillar of Social Rights. And the EU needs to train more specialists in AI, building on its long tradition of academic excellence, to create the right environment for them to work in the EU, and attract more talent from abroad.

\section{The EU AI Strategy Proposal}

The EU is still at the beginning of the process of creating a coherent strategy, but it has established some ground rules and markers already. It has decided that its strategy must take account of innovation, infrastructure, research facilities, testing environments, financial means, ethical and legal frameworks, and adequate skills levels to invest in and deploy AI. The strategy foresees maximising the impact of investments at EU and national levels, encouraging synergies and co-operation across the EU, including on ethics, fostering the exchange of best practices, and collectively defining the way forward. "The Union will progressively increase efforts for the take-up of AI in areas of public interest, such as healthcare, transport, security and education," it has decided [32].

The EU has set its sights on completion of the Digital Single Market and its regulatory framework, including the swift adoption of the Commission proposal for a European Cybersecurity Industrial Technology and Research Competence Centre and the Network of National Coordination Centres, reinforced connectivity through spectrum co-ordination, very fast $5 \mathrm{G}$ mobile networks and optical fibres, next generation clouds, and satellite technologies [17].

The common database of health images to be developed by the Commission in the context of Horizon 2020 
will initially be dedicated to the most common forms of cancer, using AI to improve diagnosis and treatment, and it will meet all necessary regulatory, security, and ethical requirements. The way we approach AI will define the world we live in. Amid fierce global competition, a solid European framework is needed.

\section{What We Want as a Strategy for Al in Healthcare}

The EU should put in place a coherent strategy for AI in healthcare in Europe. This will mean preparing the healthcare systems in Europe so that the benefits from AI can be felt by patients, healthcare systems, and society at large. That breaks down into a series of specific tasks, outlined in this paper. It requires:

- creating EU-wide common data spaces that promote access to health data in a secure environment, with $100 \%$ of health data digitized and computable within 5 years and with robust semantic and technical interoperability;

- integrating AI into Europe's operations so as to improve clinical care, drive new therapies and treatments, and make healthcare systems more efficient;

- committing resources to development and adoption of $\mathrm{AI}$ and promoting its use, through testing innovative AI solutions in healthcare settings;

- building trust by promoting the engagement of all healthcare stakeholders to understand the technology, and training and education for patients, medical professionals, healthcare organisations, policymakers, and governments;

- guarding against any unintended consequences of its use, through appropriate policy frameworks that preserve privacy while permitting innovation, and that address data bias and promote equitable access.

On each of these points, the EU should act. If it does, the benefits for EU citizens, society, and prosperity will follow.

\section{Conclusion}

Europe stands on the brink of a huge success. If it manages to seize the opportunity that AI presents for healthcare, it can improve life for its citizens, make better use of its healthcare budgets, and enhance its competitiveness in a sector that will dominate global growth for a generation. This paper has set out the stakes: with everything to play for, policy decisions can now allow Europe to win the prize. But scientists, technologists, researchers, companies, and patients can do no more than explain and advise. It is politicians who have the decision-making power, who will influence the environment, and who will, therefore, ultimately determine whether Europe wins the prize or lets it slip through its fingers. That is why this paper is addressed to Europe's political class. We have done our bit. Now we urge you to do yours.

\section{Acknowledgements}

We would like to thank partners from "DigitalHealthEurope: Support to a Digital Health and Care Innovation initiative in the context of Digital Single Market strategy" for their input.

\section{Disclosure Statement}

The authors declare that they have no competing interests.

\section{Funding Sources}

There are no funding sources for this paper to declare.

\section{Author Contributions}

D.H. conceived, built, and drafted the article with critical input from his co-authors and MEGA+ partners.

\section{References}

AI: Power for Civilisation - and for Better Healthcare
1 Dilsizian SE, Siegel EL. Artificial intelligence in medicine and cardiac imaging: harnessing big data and advanced computing to provide personalized medical diagnosis and treatment. Curr Cardiol Rep. 2014 Jan;16(1):441.

2 Study Panel for the Future of Science and Technology, EPRS - European Parliamentary Research Service, Scientific Foresight Unit (STOA). Understanding algorithmic decision-making: Opportunities and challenges. PE 624.261 - March 2019. Available from: http://www.europarl.europa.eu/Reg-
Data/etudes/STUD/2019/624261/EPRS_ STU(2019)624261_EN.pdf.

3 Lewis N. Artificial intelligence to play key role in population health. Med Econ. 2017. Available from: http://www.medicaleconomics. com/medical-economics-blog/artificial-intelligence-play-key-role-population-health

4 American Medical Association. Augmented intelligence in health care. 2018. 18257409:PDF:7/18 Available from: https:// www.nist.gov/system/files/documents/2019/06/06/nist-ai-rfi-ama-001.pdf. 
5 Kalias B, Collier M, Fu R. 10 promising AI applications in health care. Harv Bus Rev. 2018 May 10.

6 Communication from the Commission to the European Parliament. The Council, The European Economic and Social Committee and the Committee of the Regions on enabling the digital transformation of health and care in the Digital Single Market; empowering citizens and building a healthier society. COM/2018/233 final. Cited: August 21, 2019. Available from: https://eur-lex.europa.eu/legal-content/EN/ ALL/?uri=COM: 2018:233:FIN

7 Council conclusions on innovation for the benefit of patients. (2014/C 438/06). Cited: August 21, 2019. Available from: https://eurlex.europa.eu/legal-content/EN/TXT/ $\mathrm{PDF}$ /?uri=CELEX: 52014XG1206(03)\&from $=\mathrm{EN}$

8 Faircloth B, Heskett C, Roper S. Artificial Intelligence: Six Challenges for the European Healthcare Sector, vol XX, issue 59, November 30, 2018. Available from: https://www.lek. $\mathrm{com} / \mathrm{sites} / \mathrm{default/files/insights/pdf-}$ attachments/2059-AI-Challenges-for-European-Healthcare-Sector.pdf

9 Marr B. AI That Saves Lives: The Chatbot That Can Detect A Heart Attack Using Machine Learning, Forbes 4,188 views. Dec 21, 2018. Available from: https://bernardmarr. com/default.asp? contentID $=1762$

10 Siwicki B. Magellan Health uses smartphone-based AI tech to help Hep C patients, August 12, 2019. Cited: October 18, 2019. Available from: https://www.healthcareitnews.com/news/magellan-health-usessmartphone-based-ai-tech-help-hep-c-patients

11 Muoio D. How chatbots and robots can fill healthcare's unmet needs, September 25, 2018. Cited: October 18, 2019. Available from: https://www.mobihealthnews.com/ content/how-chatbots-and-robots-can-fillhealthcares-unmet-needs

12 Keshava N. Opportunities for Data Science in the Pharmaceutical Industry - The Use of Data to Find Efficiencies in Drugs Development Can't Come Too Soon. IEEE Pulse. 2017 May-Jun;8(3):10-4.

13 Harrer S, Shah P, Antony B, Hu J. Artificial Intelligence for Clinical Trial Design. Trends Pharmacol Sci. 2019 Aug;40(8):P577-91. Available from: https://doi.org/https://doi. org/10.1016/j.tips.2019.05.005.

14 Sherlock in Health. How artificial intelligence may improve quality and efficiency, whilst reducing healthcare costs in Europe. PWC. 2017 June. Available from: https://www.pwc. de/de/gesundheitswesen-und-pharma/studie-sherlock-in-health.pdf

15 Venkatesan P. 2018 Annual Congress of the European Respiratory Society. Lancet Respir Med. 2018 Nov;6(11):P815-6.

16 Department of Health and Social Care. Health Secretary announces $£ 250$ million investment in artificial intelligence. Published: August 8, 2019. Available from: https://www.gov.uk/ government/news/health-secretary-announces-250-million-investment-in-artificial-intelligence

17 Communication from the Commission to the European Parliament, the Council, the European Economic and Social Committee and the Committee of the Regions. Mid-Term Review on the implementation of the Digital Single Market Strategy A Connected Digital Single Market for All. SWD(2017) 155 final. Cited: August 21, 2019. Available from: https://eur-lex.europa.eu/content/news/digital_market.html

18 Powles J, Hodson H. Google DeepMind and healthcare in an age of algorithms. Health Technol (Berl). 2017;7(4):351-67.

19 Bughin J, Hazan E, Ramaswamy S, Chui M, Allas T, Dahlström P, Henke N, Trench M. Artificial intelligence the next digital frontier?. McKinsey \& Company. McKinsey Global Institute. 2017;47. Available from: https://www.mckinsey.com/ /media/McKinsey/Industries/Advanced\%20Electronics/ Our\%20Insights/How\%20artificial\%20intelligence $\% 20$ can $\% 20$ deliver $\% 20$ real $\% 20$ value $\% 20$ to $\% 20$ companies/MGI-ArtificialIntelligence-Discussion-paper.ashx

20 Branca F, Nikogosian H, Lobstein T, editors. The challenge of obesity in the WHO European Region and the strategies for response: summary. Copenhagen: World Health Organization; 2007.

21 Skaane P, Bandos AI, Gullien R, Eben EB, Ekseth U, Haakenaasen U, et al. Comparison of digital mammography alone and digital mammography plus tomosynthesis in a population-based screening program. Radiology. 2013 Apr;267(1):47-56.

22 Patel TA, Puppala M, Ogunti RO, Ensor JE, $\mathrm{He} \mathrm{T}$, Shewale JB, et al. Correlating mammographic and pathologic findings in clinical decision support using natural language processing and data mining methods. Cancer. 2017 Jan;123(1):114-21.

$23 \mathrm{He}$ KY, Ge D, He MM. Big Data Analytics for Genomic Medicine. Int J Mol Sci. 2017 Feb; 18(2):412

24 Consultative Committee of the Convention for the Protection of Individuals with Regard to Automatic Processing of Personal Data. Report on Artificial Intelligence; Artificial Intelligence and Data Protection: Challenges and Possible Remedies. T-PD(2018)09Rev Available from: https://rm.coe.int/artificialintelligence-and-data-protection-challengesand-possible-re/168091f8a6

25 Pifer R. How AI could shape the health tech landscape in 2019. HealthcareDrive. Published: January 10, 2019. Available from: https://www.healthcaredive.com/news/howai-could-shape-the-health-tech-landscapein-2019/545461/

26 Horgan D, Bernini C, Thomas PPM, Morre SA: Cooperating on data: the missing element in bringing real innovation to Europe's healthcare system. Public Health Genomics. 2019. DOI: $10.1159 / 000503296$.
27 Innovative Solutions for Research in Healthcare. Developing a novel approach to deliver better precision medicine in Europe. The EMA standpoint. Cited: August 21, 2019. Available from: http://www.europarl.europa. eu/cmsdata/159164/Guido\%20Rasi.pdf

28 Wood AR, Esko T, Yang J, Vedantam S, Pers $\mathrm{TH}$, Gustafsson S, et al.; Electronic Medical Records and Genomics (eMEMERGEGE) Consortium; MIGen Consortium; PAGEGE Consortium; LifeLines Cohort Study. Defining the role of common variation in the genomic and biological architecture of adult human height. Nat Genet. 2014 Nov;46(11): 1173-86.

29 COMPARE is a multidisciplinary research network that has the common vision to become the enabling analytical framework and globally linked data and information sharing platform for the rapid identification, containment and mitigation of emerging infectious diseases and foodborne outbreaks (http:// www.compare-europe.eu/about)

30 Correlates protection, determinants of outcome, and clinical management (http://www. evident-project.eu/)

31 The Lancet Public Health. Next generation public health: towards precision and fairness. Lancet Public Health. 2019 May;4(5):PE209.

32 Communication from the Commission to the European Parliament, the European Council, the European Economic and Social Committee and the Committee of the Regions on Artificial Intelligencefor Europe.SWD/2018/137 final. Cited: September 23, 2019. Available from: https://eur-lex.europa.eu/legal-content / EN / T X T / P D F / ? uri = CELEX: 52018DC0237\&from $=\mathrm{GA}$

33 COCIR:The European Coordination Committee of the Radiological, Electromedical and Healthcare IT Industry. Artificial Intelligence in Healthcare - April 2019. Cited: September 23, 2019. Available here: https://www. cocir.org/uploads/media/COCIR_White_ Paper_on_AI_in_Healthcare.pdf

34 Irish Statute Book. S.I. No. 314/2018 - Data Protection Act 2018 (Section 36(2)) (Health Research) Regulations 2018. Available from: http://www.irishstatutebook.ie/eli/2018/ si $/ 314 /$ made/en/print

35 EU Cloud in Health Advisory Council. Enable data-driven healthcare \& research for citizen benefit while protecting patient privacy (Jan. 10, 2018). Available here: http://cloudinhealthadvisorycouncil.eu/papers/enable-data-driven-healthcare-research-for-citizenbenet-while-protecting-patient-privacy/

36 Official Journal of the European Union. Regulation 2017/745 of the European Parliament and of the Council of 5 April 2017 on medical devices, amending Directive 2001/83/EC, Regulation (EC) No 178/2002 and Regulation (EC) No 1223/2009 and repealing Council Directives 90/385/EEC and 93/42/EEC. Available from: https://eur-lex.europa.eu/legalcontent/EN/TXT/PDF/? uri=CELEX: 32017R0745 
37 High-Level Expert Group on Artificial Intelligence. Ethics guidelines for trustworthy AI. Published: April 8, 2019. Available from: https://ec.europa.eu/digital-single-market/ en/news/ethics-guidelines-trustworthy-ai

38 Official Journal of the European Union. Civil Law Rules on Robotics (2018/C 252/25). European Parliament resolution of 16 February 2017 with recommendations to the Commission on Civil Law Rules on Robotics (2015/2103(INL)). Available from: https:// eur-lex.europa.eu/legal-content/EN/TXT/?u $\mathrm{ri}=$ uriserv:OJ.C_.2018.252.01.0239.01. ENG\&toc=OJ:C:2018:252:TOC

39 Bezemer T, de Groot MC, Blasse E, Ten Berg MJ, Kappen TH, Bredenoord AL, et al. A Human(e) Factor in Clinical Decision Support Systems. J Med Internet Res. 2019 Mar; 21(3):e11732.

40 FDA US Food \& Drug Administration. Proposed Regulatory Framework for Modifications to Artificial Intelligence/Machine Learning (AI/ML)-Based Software as a Medical Device (SaMD) - Discussion Paper and Request for Feedback. Cited: September 23, 2019. Available from: https://www.fda.gov/ media/122535/download

41 OECD. Artificial Intelligence in Society. Paris: OECD Publishing; 2019. https://doi. org/10.1787/eedfee77-.

42 Hoffman D, Masucci R. Intel's AI Privacy Policy White Paper. October 2018. Available from: https://blogs.intel.com/policy/files/ 2018/10/Intels-AI-Privacy-Policy-White-Paper-2018.pdf

43 Eurobarometer surveys on 'Digital health literacy' and 'Data protection'; Taylor Wessing.

44 EFPIA European Federation of Pharmaceutical Industries and Associations. Oncology data landscape in Europe. July 2018. Available from: https://www.efpia.eu/media/412317/ efpia-onco-data-landscape-6-solutions.pdf

45 Final Report and Action Plan from the European Commission Expert Group on FAIR Data - Turning FAIR into reality. November 2018. Available from: https://ec.europa.eu/ info/sites/info/files/turning_fair_into_reality_0.pdf

46 Official Journal of the European Union. Regulation (EU) 2016/679 of the European Par- liament and of the Council of 27 April 2016 on the protection of natural persons with regard to the processing of personal data and on the free movement of such data, and repealing Directive 95/46/EC (General Data Protection Regulation). Available from: https://eur-lex. europa.eu/legal-content/EN/TXT/PDF/ ?uri=CELEX:32016R0679Official Journal of the

47 Act on the Secondary Use of Health and Social Data. Available from: https://stm.fi/documents/1271139/1365571/The+Act+on+the+ Secondary + Use + of + Health + and + Social + Da ta/a2bca08c-d067-3e54-45d1-18096de0ed76/ The+Act+on+the+Secondary+Use+of + Heal th+and+Social+Data.pdf

48 World Health Organization. WHO releases first guideline on digital health. April 17, 2019. Available from: https://www.who.int/ news-room/detail/17-04-2019-who-releasesfirst-guideline-on-digital-health-interventions

49 Char DS, Shah NH, Magnus D. Implementing Machine Learning in Health Care - Addressing Ethical Challenges. N Engl J Med. 2018 Mar;378(11):981-3.

50 Feng A, Wu S. The Myth of the Impartial Machine. Parametric Press, Issues 01 Science +Society. Available from: https://parametric. press/issue-01/the-myth-of-the-impartialmachine/

51 Communication from the Commission to the European Parliament, the European Council, the Council, the European Economic and Social Committee and the Committee of the Regions. Coordinated plan on artificial intelligence $\operatorname{COM}(2018) 795$ final. Cited: September 23, 2019. Available from: https://eur-lex. europa.eu/legal-content/EN/TXT/ HTML/?uri=CELEX: 52018DC0795\&from= DA

52 Commission publishes evaluation reports on EU rules on machinery safety and product liability. May7, 2018. Available from: https:// ec.europa.eu/growth/content/commissionpublishes-evaluation-reports-eu-rules-machinery-safety-and-product-liability_en

53 Milano M. The digital skills gap is widening fast. Here's how to bridge it. The World Economic Forum. March 12, 2019. Available from: https://www.weforum.org/agenda/ 2019/03/the-digital-skills-gap-is-wideningfast-heres-how-to-bridge-it/

54 Horgan D, van Kranen HJ, Morré SA. Optimising SME Potential in Modern Healthcare Systems: Challenges, Opportunities and Policy Recommendations. Public Health Genomics. 2018;21(1-2):1-17.

55 Health at a Glance: Europe 2016 - State of Health in the EU Cycle. Cited: July 28, 2018. Available from: https://ec.europa.eu/docsroom/documents/26563/attachments/1/ translations/en/.../pdf

56 Communication from the Commission to the European Parliament, the European Council, the Council, the European Economic and Social Committee and the Committee of the Regions. A renewed European Agenda for Research and Innovation - Europe's chance to shape its future: the European Commission's contribution to the Informal EU Leaders' meeting on innovation in Sofia on 16 May 2018. Cited: July 28, 2018. Available from: https://ec.europa.eu/info/sites/info/files/ com-2018-306-a-renewed-europeanagenda-_for_research-and-innovation may_2018_en_0.pdf

57 Council of the European Union: Conclusions on "Think Small First - A Small Business Act for Europe". Cited: July 30, 2018. Available from: www.consilium.europa.eu/uedocs/cms Data/docs/pressdata/en/intm/104403.pdf

58 Communication from the Commission to the European Parliament, the Council, the European Economic and Social Committee and the Committee of the Regions. "Small Business, Big World - a new partnership to help SMEs seize global opportunities." Cited: July 30, 2018. Available from: https://eur-lex.europa.eu/legal-content/EN/ALL/?uri=CELEX: 52011DC0702

59 Hughes R. DeepMind: First major AI patent filings revealed. June 7, 2018. Available from: https://ipkitten.blogspot.com/2018/06/deepmind-first-major-ai-patent-filings.html

60 Declaration Of Cooperation. 'Towards access to at least 1 million sequenced genomes in the European Union by 2022'. Cited: August 21, 2019. Available from: https://www.euapm.eu/ pdf/EAPM_Declaration_Genome.pdf 